\title{
Tips from the Trenches
}

\section{Anterior shoulder reductions revisited}

\author{
Marianne McKinley, BA, MD
}

$\mathbf{P}$ atients with uncomplicated anterior shoulder dislocations often spend a prolonged period in pain and in the emergency department (ED) because of the frequent need for procedural sedation. These considerations, together with variable success rates using a variety of methods, prompted an approach that capitalizes on the strengths and minimizes the limitations of previously described methods.

The following steps describe the procedure that I have found to be most helpful:

1.Ease the patient into a sitting position if he or she can tolerate it (I have had to use single drug analgesia to accomplish this in a minority of patients).

2. Ask an assistant to stand behind the patient and stabilize him or her at the shoulders (no sheets required).

3. With the affected arm flexed at the elbow and adducted, place one hand at the base of the forearm and use the other hand to support the wrist.

4. Slowly apply downward traction by pressing down on the forearm proximally. Gravity is effectively used this

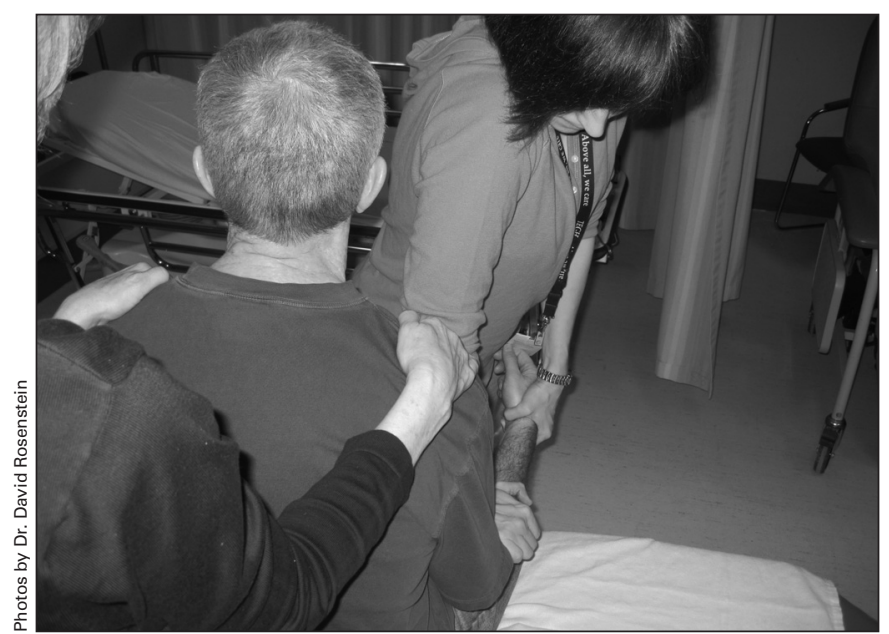

Fig. 1. Downward pressure at the elbow using arm strength and body weight. way and most people appear to have less pain at this point. This may be because the humeral head is disimpacted. This seems to be more difficult to accomplish with the patient in a supine position. Also, it's easier to apply greater force on the arm by using one's body weight than by pulling on it against gravity (Fig. 1).

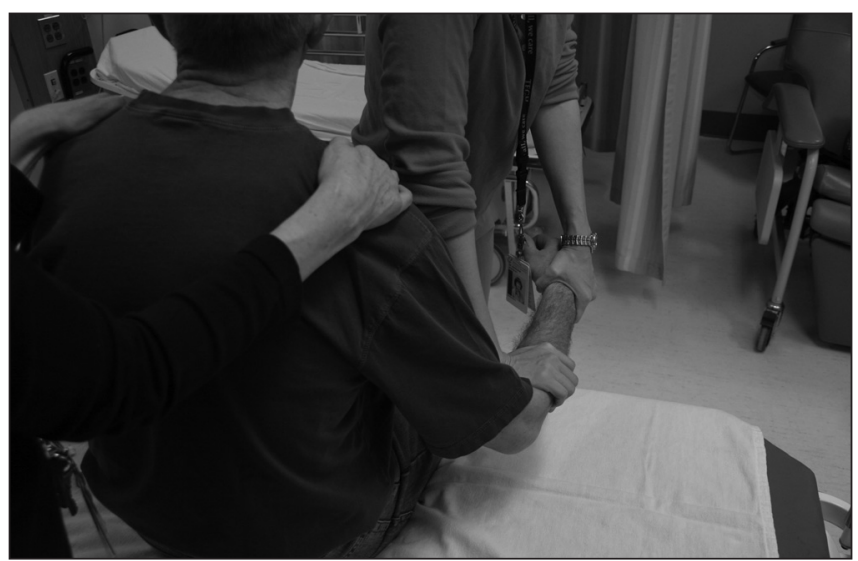

Fig. 2. Levering the arm into slight abduction while maintaining downward pressure.

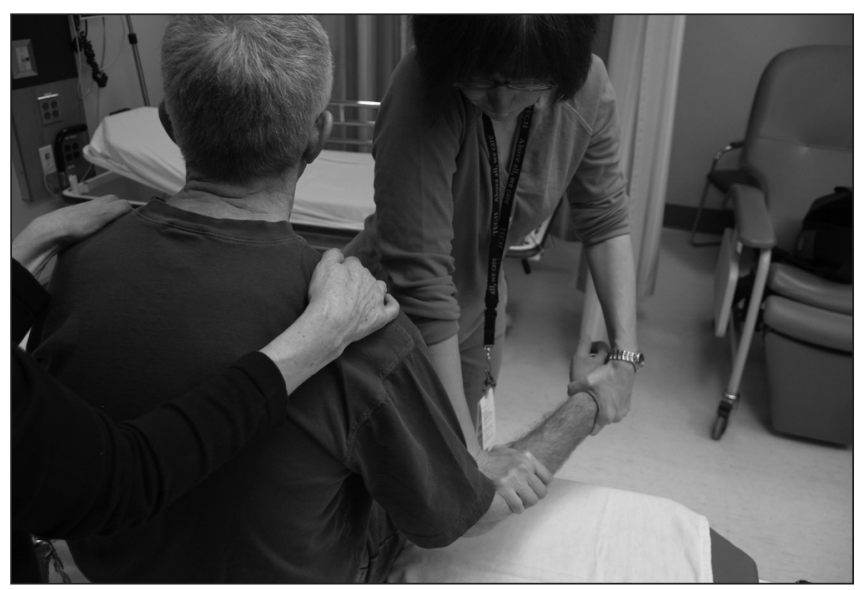

Fig. 3. Slight eversion may also be helpful as long as traction is maintained.

From the Department of Emergency Medicine, Toronto East General Hospital, Toronto, Ont.

Submitted Jan. 13, 2009; Revised Feb. 10, 2009; Accepted Feb. 15, 2009

This article has not been peer reviewed.

CJEM 2009;11(4):385-6 

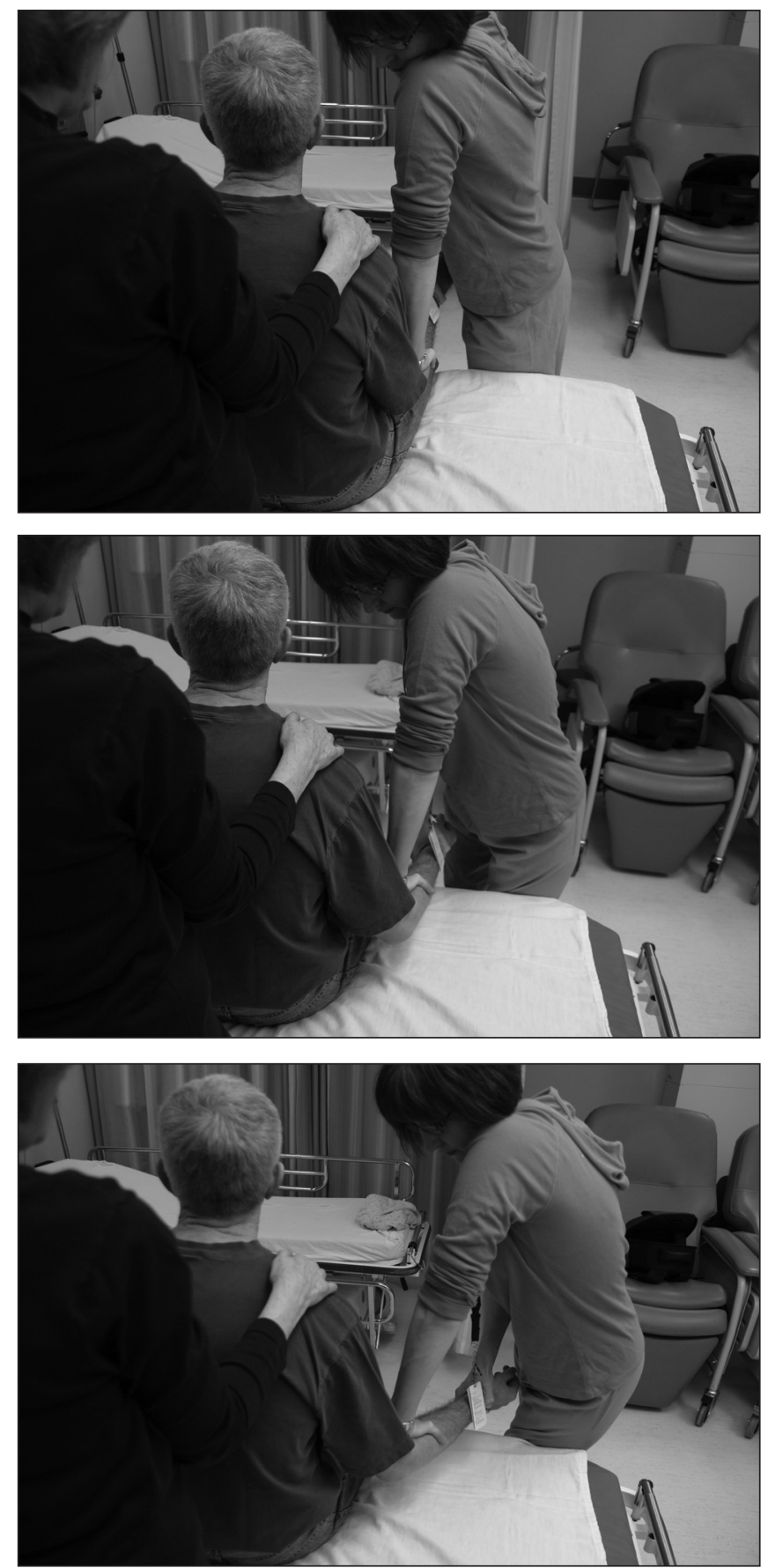

Fig. 4. Procedure in reverse position.
5. While maintaining the downward traction, slowly abduct the arm (Fig. 2).

6. If the shoulder isn't reduced by this point, then it may also be necessary to slowly evert the arm at the elbow using the hand at the wrist (Fig. 3).

7. Firm downward traction must be maintained if combined with abduction and eversion. Intuitively, this is less likely to result in a secondary fracture than everting the arm alone. (See Fig. 4 for the procedure in the reverse position.)

Thus far, I have applied this method to adult patients with a radiologically confirmed anterior shoulder dislocation. Patients with dislocations that occurred more than 24 hours before presentation have not been good candidates for this method and have invariably required procedural sedation as a minimum. Patients with new as well as recurrent dislocations have been successfully reduced. I have not applied this method to patients with significant other shoulder pathology or those requiring more urgent care. The patient must be able to tolerate a sitting position.

This method has resulted in a very high rate of success and patient satisfaction, and reduces the time patients spend in the ED, as well as the resources used.

Competing interests: None declared.

Keywords: anterior, shoulder, dislocation, reduction

Correspondence to: Dr. Marianne McKinley, Department of Emergency Medicine, Toronto East General Hospital, 825 Coxwell Ave., Toronto ON M4C 3E7; marianne.mckinley@sympatico.ca 